Purdue University

Purdue e-Pubs

ASEE IL-IN Section Conference

\title{
Can an Engineering Competition Catalyze Curriculum Innovation?
}

\author{
Ryder C. Winck \\ Rose-Hulman Institute of Technology \\ Alan Jern \\ Rose-Hulman Institute of Technology \\ Carlotta Berry \\ Rose-Hulman Institute of Technology \\ Yosi Shibberu \\ Rose-Hulman Institute of Technology
}

Follow this and additional works at: https://docs.lib.purdue.edu/aseeil-insectionconference

Winck, Ryder C.; Jern, Alan; Berry, Carlotta; and Shibberu, Yosi, "Can an Engineering Competition Catalyze Curriculum Innovation?" (2018). ASEE IL-IN Section Conference. 7.

https://docs.lib.purdue.edu/aseeil-insectionconference/2018/innov/7

This document has been made available through Purdue e-Pubs, a service of the Purdue University Libraries. Please contact epubs@purdue.edu for additional information. 


\title{
Can an Engineering Competition Catalyze Curriculum Innovation?
}

\author{
Ryder C. Winck \\ Mechanical Engineering Department \\ Rose-Hulman Institute of Technology \\ Terre Haute, IN, USA \\ Carlotta Berry \\ Electrical and Computer Engineering Department \\ Rose-Hulman Institute of Technology \\ Terre Haute, IN, USA
}

\author{
Alan Jern \\ Humanities and Social Sciences Department \\ Rose-Hulman Institute of Technology \\ Terre Haute, IN, USA \\ Yosi Shibberu \\ Mathematics Department \\ Rose-Hulman Institute of Technology \\ Terre Haute, IN, USA
}

\begin{abstract}
This article describes the ongoing efforts of a multidisciplinary group of faculty at an undergraduate institution to form a team and compete in the IBM AI XPRIZE competition. We describe the advantages and disadvantages of faculty participation in major engineering competitions over more traditional professional activities at undergraduate engineering institutions. Our discussion is focused on the benefits to three major groups: undergraduate students, faculty, and academic institutions. We use examples from our one year of experience in the competition and from the literature to illustrate these benefits. Already observed benefits from the competition include increased student engagement, development and introduction of a new minor in cognitive science, the purchase of a state-of-the-art robot and a deep learning server, enhanced multidisciplinary collaboration among faculty, and heightened awareness among administrators of the growing importance of artificial intelligence (AI) technologies. Results of a student survey regarding their involvement in with the team are presented.
\end{abstract}

Introduction:

Major engineering competitions, such as the IBM Watson AI XPRIZE competition, provide unique benefits to undergraduate engineering education. Similar past competitions include the DARPA grand challenges, the Ansari XPRIZE, the Netflix Prize, and Robocup [1][5]. While there is plenty of variation among these competitions, there are some general characteristics that make them different from other types of opportunities [5]. Competitions provide well-defined goals and criteria for success. They present problems that are highly valuable to society, and the prize money validates the value of the work. National and international competitions create publicity and excitement that can result in greater interactions across diverse backgrounds. Competitions present fixed deadlines that spur effort and provide outside assessment of accomplishments. Finally, competitions tend to be multidisciplinary and exist on the boundary between fundamental research and engineering practice [6]-[7].

Our AI XPRIZE challenge is to make it possible for a novice user to collaborate with a robot by using only verbal commands and hand gestures. Our challenge requires a multidisciplinary approach involving both the technical aspects of robotics and the challenge of human-machine communication and understanding. Much can be achieved by bringing people from multiple backgrounds together to work on a common problem. In contrast to preparing 
lesson plans or submitting a grant proposal, the AI XPRIZE competition has forced our group to focus on achieving immediate results, and thus it has accelerated our collaboration efforts.

We begin by providing some background on the IBM AI XPRIZE competition. Then we briefly describe the benefits that major engineering competitions provide to undergraduate students, faculty, and academic institutions.

\section{Background:}

During the Summer 2016, a group of 7 faculty from several disciplines (computer science, mathematics, psychology, mechanical, biomedical, and electrical engineering) got together to study the intersection of Artificial Intelligence (AI), Robotics, and Big Data. The product of this 8 week research/reading group was the creation of a learning community on human-robot collaboration. During the fall of 2016, we entered the IBM Watson AI XPRIZE competition. Graduate and undergraduate students were invited to join the research group to help us create a minimum viable product as part of our AI XPRIZE competition plan.

The AI XPRIZE "is a $\$ 5$ million AI and cognitive computing competition challenging teams globally to develop and demonstrate how humans can collaborate with powerful AI technologies to tackle the world's grand challenges" [1]. The competition will last for 4 years with teams competing each year to stay in the competition based on their progress.

The long-term technical goal of our project is to allow a human and robot arm to seamlessly collaborate to achieve a task such as unloading groceries, tying a knot, soldering PCBs, assembling IKEA furniture or playing with toy blocks. Specifically, we are focusing on enabling a robot to respond to human gestures and verbal commands. Achieving this goal will require the robot to detect human action and infer human intent. This topic is particularly important at the moment for two major technical reasons. First, we are witnessing the migration of robots from working alone inside cages in factories to safely working in the same space alongside people, both in manufacturing and in social settings. Second, improvements in artificial intelligence enable robots to make decisions based on the mountains of data created by low cost sensors.

In addition to solving the technical challenges in the competition, we are using the AI XPRIZE as a means to provide unique educational opportunities and as a catalyst for curriculum development and faculty interaction. In the following three sections we will describe how competitions benefit students, faculty and academic institutions.

Benefits to students:

Major engineering competitions, such as the AI XPRIZE competition, provide a unique experience for undergraduate students in comparison with internships, pure academic research, and traditional student competitions, such as Formula SAE or AIAA Design Build Fly. Unlike traditional student competitions, participation in the AI XPRIZE competition is open to anyone, not just students. This opens the door for networking and learning across age and experience. It also provides students the opportunity to compare their efforts against working engineers. Rose- 
Hulman is a small, primarily undergraduate engineering institution. For a school like ours, the AI XPRIZE competition provides an avenue for students to see the real world application of the concepts presented in their course work. In fact, this problem-based learning allows them to actually extend their learning beyond what they would be exposed to in the classroom.

Unlike traditional student competitions and most internship experiences, the work required in major engineering competitions is on the cutting edge of the engineering disciplines involved. These type of challenges can be inspiring and the skills learned will be in high demand. The opportunity to apply cutting edge solutions to major problems has been shown to be a major motivating factor for students [8]. Chew et al. states that not only is it necessary to use competitions to motivate students to pursue careers in engineering but also to educate and help them appreciate engineering design and development [9]. Their work indicated that students were more interested in participating in the competition than actually receiving prizes.

Unlike pure academic research, these competitions require demonstration of an engineered solution. The focus is more on demonstrating a solution to a well-defined problem than making fundamental knowledge contributions. This makes it easier to engage undergraduates in meaningful ways. For example, in our project, we need to track human motion for sensing human gestures. This is a problem with known solutions in academic literature, but these solutions have not yet made their way into common engineering practice. The undergraduate students on our team were able to apply recently developed solutions to this problem. Kaiser and Troxell state that competitions have many benefits to undergraduate engineering students including innovative thinking, motivation, and professional development [10].

Finally, the AI XPRIZE and other similar competitions are highly multidisciplinary. This allows undergraduate students to see clear connections between their chosen discipline and other related disciplines. Manseur states that student learning in competitions typically comes in the form of multidisciplinary teamwork [11]. This is because engineering competitions involve the integration of knowledge from several engineering disciplines. It is very rare that an entry-level engineer or scientist will only work within one discipline, so it is important to be able to communicate and work with people from other disciplines.

The 15 students who participated in the competition during the 2016 school year were asked to complete a survey in order to obtain their feedback on this experience. Ten of the students completed the confidential and anonymous survey. The students surveyed were from the computer science and software engineering, electrical and computer engineering, mechanical engineering, and mathematics departments. The questions posed were:

- "What were your reasons for wanting to participate in the project?"

- "What have you learned during your experience on the project?"

- "What do you still hope to get out of the experience?"

The results indicated that nine joined the project due to interest in and a desire to gain experience with robotics and/or artificial intelligence. One student stated, "This project seemed the most interesting of anything I'd found when searching for jobs this summer. I'd written a research paper on IBM Watson in the past, and I wanted to learn more about how one would 
accomplish training an AI to act as human-like as possible". One student also explained the benefits with the following response, "To get a project outside of school to see if I wanted to work with robots and make myself more marketable to employers". Three students stated that they have learned how to conduct open ended research that will require iteration in the design process. One stated a better understanding of the technical concepts of AI with the comment, "So far, I've learned just how much is needed to be done before an AI can actually be trained. There's so much data that needs to be collected, and the methods of collecting the data need to be properly designed and set up before anything can happen." In addition, four students mentioned that they appreciated that testing, debugging and data collection is important and takes longer than expected. Students still hope to learn more about machine learning and use of robotic software tools based upon the following sample response, "I'm excited to see what happens once enough data is collected. I can't quite see yet how all of the data will come together in order to have the AI achieve tasks. Once this part of the project is achieved, and the AI can complete tasks based on the data we will have collected, I think that will be the most amazing experience of the project to witness." In addition, one student expressed a desire to eventually publish the results of their work. Therefore, we identified the following benefits to students of participating in the competition, based on their survey responses:

- Getting practical technical experience in AI, robotics, and research;

- Conducting work outside of traditional school work;

- Learning more about the design process, testing, and debugging;

- Practicing teamwork, communication, as well as self-direction.

Benefits to faculty:

In addition to teaching, most faculty are expected to perform other professional activities such as research, consulting, curriculum development, and K-12 and community outreach activities. How does faculty participation in competitions compare to such professional activities?

Competitions are a great way to jump-start innovations. In our case, while the AI XPRIZE competition was not the catalyst that formed our team, the competition quickly focused our efforts and gave us a framework that we could use to efficiently mobilize and utilize the resources at our institution. The fixed-deadline of the competition made the benefits of collaboration more immediate. The ME department purchased a state-of-the-art collaborative robot (the Sawyer Robot), and the ME, Math, and CSSE departments pooled resources to help fund the purchase of a Deep Learning server. The ECE department and Robotics minor program have also made robots available for use in the project. The utility of such investments in equipment is magnified when the equipment is used jointly in focused projects involving faculty from multiple departments. Faculty can share their expertise to make the most efficient use of such resources.

The time pressures created by competitions encourage focus and a problem-solving, results orientation [6]. This is in contrast to the open-ended exploration that is typical in academic research or to professional consulting, which has less academic freedom and flexibility.

Competitions can also provide new avenues to fund faculty work beyond traditional grant mechanisms . For example, many of the teams competing in the DARPA Grand Challenge were 
able to find corporate sponsors for their work [12]. In our competition, IBM has given free access to competing teams to IBM Watson AI resources. We have also been able to connect more closely with our school's administration and will be able to leverage alumni and corporate connections in addition to traditional funding avenues. Two members of the team have already received internal research grants from the Dean for this project.

Our work on the AI XPRIZE has also benefitted faculty by promoting interactions across disciplines. These benefits are a direct consequence of the multidisciplinary nature of the competition. In spite of the fact that many modern-day science and engineering challenges are fundamentally multidisciplinary in nature, Rose-Hulman, like many academic institutions, is still organized into discrete departments based on historical disciplinary boundaries [13]. As a result, even though many faculty members support multidisciplinary programs, there are few institutional incentives to develop these programs, and few opportunities for faculty members to develop professional relationships with faculty outside of their own departments. By contrast, major engineering competitions create high-profile, immediate incentives for faculty from different departments to work together. As noted earlier, our work has brought together 7 faculty members with disparate expertise from 6 different departments. These faculty have been consistently involved in the project and as of now the team has not change although there may be additions as the project advances.

Major engineering competitions provide unique collaborative benefits relative to multidisciplinary research efforts. In multidisciplinary research, faculty from different departments typically collaborate on a grant. However, once the grant is received, the incentives for collaboration are reduced, and faculty can be at odds over the direction of the research, each one wanting to focus on the work in their own field. During competitions, however, we have observed that faculty collaborations are less likely to be stymied by discipline specific difference as faculty have a concrete project to guide them with deadlines that focus efforts. In addition, the external assessment of competitions encourages faculty to maintain a common goal and sustain professional relationships.

The connections developed from working as a team in a competition can also lead to further collaboration in research efforts and curriculum development. In our case, the AI XPRIZE competition has sparked discussions on how AI and machine learning courses might be coordinated with robotics courses. These collaborative efforts not only benefit the faculty, but they also provide direct benefits to institutions.

Benefits to academic institutions:

Multidisciplinary curriculum development is challenging because faculty have different backgrounds and so do not have a common framework to facilitate collaborations. Often, not enough effort is spent understanding and exploring the different perspectives possible. When a multidisciplinary team of faculty participate in a competition, the competition provides a compelling common framework that facilitates multidisciplinary collaborations. These new professional relationships have the opportunity to lead to new, and lasting, multidisciplinary curricular developments. We believe our institution will ultimately benefit from the bridges between departments created by the multidisciplinary teamwork required by our participation in 
the AI XPRIZE competition. In this section, we describe several examples of such developments that are either enhanced or were made possible by our work on the AI XPRIZE.

First, Rose-Hulman currently offers two multidisciplinary minors related to work on the AI XPRIZE. The first is a minor in robotics. This program has been in existence for the last 8 years, and several members of the robotics faculty are also members of the AI XPRIZE research faculty. The multidisciplinary minor in robotics has proven to be a great recruiting and marketing tool for the school. In addition, potential employers have requested to hire graduates with this minor due to the unique multidisciplinary skillset students gain. Rose-Hulman also recently approved a new minor in cognitive science, a field that draws on several disciplines relevant to the AI XPRIZE, including AI, psychology, and neuroscience. The minor was developed by several members of the AI XPRIZE team. Although the development of these minors did not spawn from work on the prize, the minor programs and the AI XPRIZE effort support one another. Namely, student work on the AI XPRIZE will lead to greater interest in robotics and cognitive science, and will increase participation in the minors. For students already enrolled in the minor programs, work on the AI XPRIZE will help them to draw stronger connections between robotics, cognitive science, AI, human-robot collaboration, and related fields. This example illustrates how enthusiasm about prizes like the AI XPRIZE can promote and support related existing multidisciplinary programs on campus.

Second, one of the faculty members in our group (psychology), has been motivated to pursue a new course that combines psychology and robotics. Similar courses have been offered at other institutions [14]-[16]. What these courses have in common is that they use robots to teach students to think more mechanistically about human behavior and cognition. For example, in some courses, students were provided with pre-programmed robots. They then observed the robots' behavior and performed experiments to infer the rules driving their behavior. Courses like these draw direct links between engineering and social science by framing psychology and cognitive science as an attempt to reverse-engineer the mind. Many of these courses were based on the book "Vehicles" by Braitenberg, which describes a series of simple robots that exhibit animal-like behaviors such as love, fear, aggression, and exploration [17].

Before getting involved in the AI XPRIZE, the psychology faculty member in our group had been highly interested in developing a course like this but he did not have the relevant robotics expertise nor sufficient contact with potential collaborators in the robotics faculty to pursue it. Working on this project has set the stage for him to seriously pursue this course. This example illustrates how the professional contacts that emerge through work on a prize like the AI XPRIZE can have lasting influence well beyond the prize itself.

Kumar describes the advantages of using robotics to teach AI concepts [17]. According to Kumar, robots are more engaging for students than software alone. Robots promote hands-on learning and testing is in a physical rather than virtual environment. The AI XPRIZE competition has laid the foundations for strong professional relationships between departments. We are exploring the possibility of coordinating multi-departmental courses and student projects in robotics, AI, and machine/deep learning. This will require sharing of resources and expertise. 
In addition to multidisciplinary curriculum development, competitions also provide positive publicity for institutions [6] and new avenues through which to connect with alumni. As can be observed in sports, competitions create compelling stories and generate considerable public interest. Communities have a strong desire to see their members succeed in competitions. Competitions can create a sense of ownership and belonging in a community. We are currently at a very early stage in the competition, but already have growing interest in our activities. We have already achieved increased interaction between faculty and administrators through the competition. The team has received two internal grants and been in contact with institutional development to discuss strategies to engage businesses and alumni. Progressing in the competition, we believe, will increase awareness of the our work among the school community, alumni and potential corporate sponsors. In addition, the competition provides opportunities for publicity to the broader public [6], which can be especially beneficial to smaller schools, such as Rose-Hulman.

Conclusions and future work:

Based upon our own early experiences in the AI XPRIZE competition and the cited research, this paper describes the benefits to students, faculty and academic institutions resulting from participation in a faculty led competition. Benefits to the students include project-based learning on the cutting-edge of their disciplines. The benefits to the faculty include the creation of a mechanism to focus collaborative efforts and develop professional relationships across multiple disciplines. The benefits to academic institutions include enhancement of the existing curriculum and increased positive publicity.

Currently, students have been recruited to continue working this summer. So far this group has created an online simulation environment and in-lab instrumented testbed to collect data on human communication for human-robot collaboration. In order to continue assessing the benefits to the respective groups, there will be a focus group by the institute and additional online surveys. Assessment of the team effectiveness will be provided by execution of the competition test suite, feedback from the XPRIZE program committee, and group processing.

\section{Acknowledgments:}

The authors would like to thank Michael Wollowski, David Voltmer and Alan Chiu and the student team for their work on AI XPRIZE competition. They would also like to thank RoseHulman Institute of Technology for providing resources supporting this work.

\section{References:}

[1] IBM Watson AI XPRIZE, http://ai.XPRIZE.org/about/overview.

[2] M. Montemerlo, S. Thrun, H. Dahlkamp, D. Stavens, and S. Strohband, "Winning the DARPA Grand Challenge with an AI robot." AAAI National Conference on Artificial Intelligence, vol. 1, pp. 982-987, 2006.

[3] L. Kay, "The effect of inducement prizes on innovation: evidence from the Ansari XPRIZE and the Northrop Grumman Lunar Lander Challenge." $R \& D$ Management, vol. 41, pp. 360-377, 2011. 
[4] Bennett, J., \& Lanning, S., "The Netflix prize." Proceedings of KDD cup and Workshop, Aug. 2007.

[5] H. Kitano, M. Asada, Y. Kuniyoshi, I. Noda, E. Osawa, and H. Matsubara, "RoboCup: A challenge problem for AI. AI magazine." AI Magazine, vol. 18, pp. 73-85, 1997.

[6] E.Wagner, "Why Prize? The Surprising Resurgence of Prizes to Stimulate Innovation," Research-Technology Management, vol. 54, pp. 32-36, 2011.

[7] A. Welch and D. Huffman, "The effect of robotics competitions on high school students' attitudes toward science." School Science and Mathematics, vol. 111, pp. 416-424, 2011.

[8] I.M. Verner, S. Waks, "Educational features of robot contests: the RoboCup-98 survey." Advanced Robotics, vol. 14, pp. 65-74, 1999.

[9] M.T. Chew, S. Demidenko, C. Messom, and G.S. Gupta, "Robotics competitions in engineering education." IEEE International Conference on Autonomous Robots and Agents, Feb. 2009.

[10] C. Kaiser and W. Troxell, "Student design competitions in undergraduate engineering education." IEEE Annual Conference Frontiers in Education, Oct. 2005.

[11] R. Manseur, "Hardware competitions in engineering education." IEEE Annual Conference Frontiers in Education, Oct. 2000.

[12] S. Thrun, "A personal account of the development of Stanley, the robot that won the DARPA Grand Challenge." AI Magazine, vol. 27, pp. 69-82, 2006.

[13] M.A. Collura, B. Aliane, S. Daniels, and J. Nocito-Gobel, "Development of a multidisciplinary engineering foundation spiral." ASEE Annual Conference and Exposition, 2004.

[14] T. Stafford, "The Scientific Method - Lego Robots Edition," 2010. https://mindhacks.com/2010/06/23/the-scientific-method-lego-robots-edition/

[15] B.A. Motz, M.H. Goldstein, and L.B. Smith, "Understanding Behaviour from the Ground Up: constructing robots to reveal simple mechanisms underlying complex behaviour." Psychology Learning \& Teaching, vol. 11, pp. 77-86, 2012.

[16] T. Gureckis, "Teaching Human Psychology using Open-Source Robotics." http://gureckislab.org/blog/?p=3121

[17] V. Braitenberg, Vehicles: Experiments in synthetic psychology, The MIT Press, 1986.

[18] A.N. Kumar, "Three years of using robots in an artificial intelligence course: lessons learned." Journal on Educational Resources in Computing, vol. 4, 2004. 